\title{
Response of Cantilever Model with Inertia Nonlinearity under Transverse Basal Gaussian Colored Noise Excitation
}

\author{
Bo Li, Kai Hu (D, Guoguang Jin (D), Yanyan Song, and Gen Ge \\ School of Mechanical Engineering, Tiangong University, Tianjin 300387, China \\ Correspondence should be addressed to Guoguang Jin; jinguoguang@tiangong.edu.cn
}

Received 1 August 2020; Revised 9 December 2020; Accepted 12 January 2021; Published 20 January 2021

Academic Editor: Mariano Torrisi

Copyright (c) 2021 Bo Li et al. This is an open access article distributed under the Creative Commons Attribution License, which permits unrestricted use, distribution, and reproduction in any medium, provided the original work is properly cited.

\begin{abstract}
Considering the curvature nonlinearity and longitudinal inertia nonlinearity caused by geometrical deformations, a slender inextensible cantilever beam model under transverse pedestal motion in the form of Gaussian colored noise excitation was studied. Present stochastic averaging methods cannot solve the equations of random excited oscillators that included both inertia nonlinearity and curvature nonlinearity. In order to solve this kind of equations, a modified stochastic averaging method was proposed. This method can simplify the equation to an Itô differential equation about amplitude and energy. Based on the Itô differential equation, the stationary probability density function (PDF) of the amplitude and energy and the joint PDF of the displacement and velocity were studied. The effectiveness of the proposed method was verified by numerical simulation.
\end{abstract}

\section{Introduction}

Since A. H. Nayfeh $[1,2]$ presented and analyzed a dynamical equation governing the vibration of slender uniform Euler-Bernoulli cantilever beams, the longitudinal inertia nonlinearity and the geometrical nonlinearity in the equation aroused much attention. Some basic theoretical analysis [3-7] and experimental researches $[8,9]$ on the harmonic excited cantilevers are carried out. These works have been extended into a wide range of fields such as nonlinear vibrations, bifurcations, and chaotic motions. Among the tremendous literatures, one essential and famous conclusion is that the geometrical nonlinearity will induce hardening effect, while the longitudinal inertia nonlinearity will lead to a softening effect.

During the enthusiasm of researching the dynamics of the cantilever excited by deterministic signals, some researchers explored the responses of randomly excited cantilever models. When Er et al. [10] investigated a cantilever model subject to lateral and axial excitations being Gaussian white noise, the model was treated as a linear multipledegree-of-freedom externally excited vibrating function. Feng et al. [11-14] studied responses of cantilever model with curvature nonlinearity and longitudinal inertia nonlinearity under narrow bounded noise excitation by the method of multiple scales. In Feng et al.'s literatures, curvature nonlinearity and longitudinal inertia nonlinearity were only treated as small perturbing terms rather than strongly nonlinear terms. Ge and Yan [15] studied a cantilever model with longitudinal inertia nonlinearity and the geometrical nonlinearity under basal white noise excitation. In this paper, the inertia nonlinearity term was simplified by Taylor series expansion, and thus, accuracy was reduced to some extent.

At present, the most effective tool to deal with wideband noise excited systems is the stochastic averaging method [16-19]. At first, the standard stochastic averaging method initialized by Landau and Stratonovich [16] and Khasminskii [17] is also called as the method of quasiconservative averaging. Later, Zhu and Lin $[18,19]$ rederived an approach named the stochastic averaging of energy envelope by considering the conservative part of the Wong-Zakai correction terms of the system. Zhu and Huang [20, 21] extended $\mathrm{Xu}$ and Chung's [22] method to handle strongly nonlinear oscillators with lightly linear and/or nonlinear damping subject to weakly external and/or parametric excitations of wideband random processes. Ge and $\mathrm{Li}$ [23] have simplified Zhu and Huang's method $[20,21]$ with a cost of slightly reducing the prediction accuracy. 
Although the improved energy envelope stochastic averaging methods [20-23] are effective on randomly excited oscillators with strong geometric nonlinearities, it cannot offer effective approach to solve randomly excited systems with inertia nonlinearity in the form of $\ddot{x}+\omega^{2} x+g(x)+c(x, \dot{x})=\eta(t)$, where $g(x)$ is the nonlinear stiffness term (geometric nonlinearity), $c(x, \dot{x})$ is the damping term, and $\eta(t)$ is the random excitation, but the present methods cannot deal with the oscillator in the form of $\left(1+\alpha x^{2}\right) \ddot{x}+\omega^{2} x+g(x)+$ $\alpha x \dot{x}^{2}+c(x, \dot{x})=\eta(t)$, where the $\left(1+\alpha x^{2}\right)$ is the so-called inertia nonlinearity (also called as coordinate-dependent mass in some literature). Thus, a new method is needed. We will investigate an improved stochastic averaging method to deal with the randomly excited differential equations with both inertia nonlinearity and the geometric nonlinearity. In other words, we will extend the present method into a new field.

Literatures on the cantilever model subject to noise are mostly concentrated on the narrow-banded noise [11-14] or Gaussian white noise [15]. The advantage of the white noise model over the others is that it is mathematically simple and idealistic. Compared to the white noise, Gaussian colored noise may be a more suitable mathematical model to simulate the environment behavior. As is well known [24, 25], Gaussian colored noise can be obtained by a zero-mean Gaussian white noise passing through a linear filter $\dot{\eta}=-\lambda \eta+\lambda \xi(t)$. The correlation function form is as follows:

$$
R_{i j}(\tau)= \begin{cases}\lambda_{i} D_{i} e^{\left(-\lambda_{i i}|\tau|\right)}, & i=j, \\ 0, & i \neq j,\end{cases}
$$

where $\lambda$ denotes the correlation time parameter and $\xi(t)$ denotes a Gaussian white noise with 0 mean and intensity of $2 D$. The expression of equation (1) can be also seen in $[24,25]$. If $D=1$ and $\lambda=0.1,0.2$, Figure 1 shows the diagram of the correlation function $R$. As is well known, the autocorrelation function of the white noise is $R(\tau)=E[\xi(t) \xi(t+\tau)]=$ $D \delta(\tau)$, and the $\delta(\tau)$ is the Dirac delta function. If the parameter $\lambda$ in equation (1) is infinitely large, the colored noise will decrease to be a Gaussian with noise.

In order to study the cantilever model excited by Gaussian colored noise, a stochastic averaging method which can handle the equations with inertia nonlinearity and the geometrical nonlinearity is presented. To avoid being esoteric, the deduction procedure is elaborated in Methodology. After the averaging, an Itô type stochastic differential equation together with the draft coefficient and the diffusion coefficient is presented. Subsequently, the stationary responses of the cantilever model are given. The probability density function (PDF) of the equivalent amplitude, the vibrating energy, and the joint probability density function of the displacement and velocity are obtained. Numerical simulations on the randomly excited equations are employed to verify the theoretical deductions.

\section{Model Assumptions}

A uniform inextensible cantilever was characterized by the mass per unit length $m$, the bending stiffness $E I$, length $L$, linear viscose damping coefficient $c$, and transversal

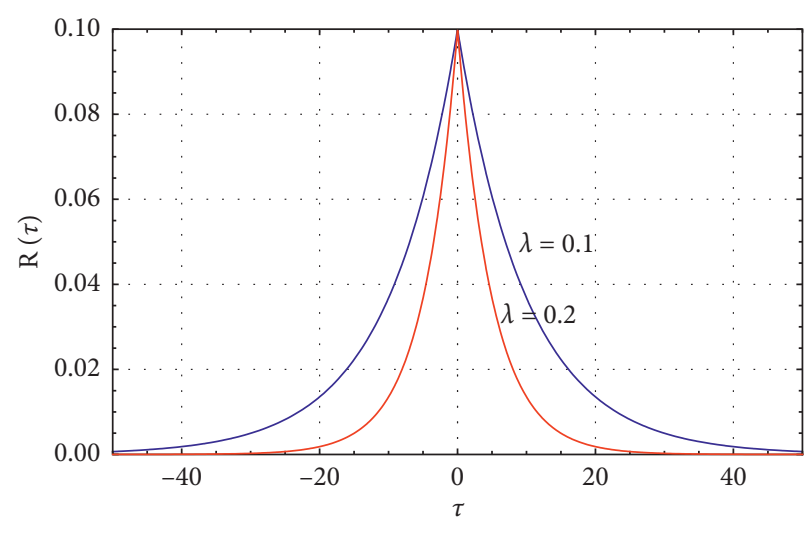

FIgURE 1: The correlation function of the colored noise.

displacement of the pedestal $h(t)$. The symbol $s$ denotes the axial spatial Lagrange coordinate, (') denotes the partial derivative with respect to $s$, and $(\cdot)$ denotes the partial derivative with respect to time $t$. Only the planar motions of the beam are concerned, and the gravitational effects are neglected. If $h(t)$ is in the form of harmonic signal and the frequency of which is near the $i$-th mode's frequency of the cantilever, the internal resonance between modes is negligible. Therefore, a well-known dynamic model governing a slender pedestal transversely excited in-extendable cantilever beam is presented as follows [15]:

$$
\begin{aligned}
& \rho A \int_{0}^{L} \phi_{i}^{2} \mathrm{~d} s \ddot{q}_{i}+c \int_{0}^{L} \phi_{i}^{2} \mathrm{~d} s \dot{q}_{i}+E I \int_{0}^{L} \phi_{i}^{\prime \prime} \mathrm{d} s q_{i}+2 E I \int_{0}^{L} \phi_{i}^{\prime \prime 2} \phi_{i}^{\prime 2} \mathrm{~d} s q_{i}^{3}+ \\
& \rho A \int_{0}^{L}\left(\int_{0}^{s} \phi_{i}^{\prime 2} \mathrm{~d} s\right)^{2} \mathrm{~d} s\left(q_{i} \dot{q}_{i}^{2}+q_{i}^{2} \ddot{q}_{i}\right)=-\rho A \int_{0}^{L} \phi_{i} \mathrm{~d} \ddot{h}(t),
\end{aligned}
$$

where $\rho$ is the density, $A$ is the cross section area, $E I$ is the bending moment, $c$ is the damping coefficient, $s$ is the coordinate along the axial, the transverse displacement of the cantilever is expressed as $w_{i}(s, t)=q_{i}(t) \phi_{i}(s), q_{i}(t)$ denotes the variable separated displacement, and $\phi_{i}(s)$ denotes the $i$ th order mode functions:

$$
\begin{aligned}
\phi_{i}(s)= & \cosh \left(\lambda_{i} s\right)-\cos \left(\lambda_{i} s\right)+\frac{\cos \left(\lambda_{i}\right)+\cosh \left(\lambda_{i}\right)}{\sin \left(\lambda_{i}\right)+\sinh \left(\lambda_{i}\right)} \\
& \cdot\left[\sin \left(\lambda_{i} s\right)-\sinh \left(\lambda_{i} s\right)\right] .
\end{aligned}
$$

The mode functions are so famous that one can find it in any textbook about vibration. Although the mode function is deduced from the linearly vibrating cantilever, it is available on nonlinear vibrations [3-10]. $\lambda_{i}$ is the roots of the transcendental frequency equation $1+\cos (\lambda) \cosh (\lambda)=0$, and the first three of them are $\lambda_{1}=1.8751, \lambda_{2}=4.6941$, and $\lambda_{3}=7.8548$. The fourth term $2 E I \int_{0}^{L} \phi_{i}^{\prime \prime 2} \phi_{i}^{\prime 2} \mathrm{~d} s q_{i}^{3}$ is a static-type cubic nonlinearity associated with the potential energy stored in bending, and the fifth term $\rho A \int_{0}^{L}\left(\int_{0}^{s} \phi_{i}^{\prime 2} \mathrm{~d} s\right)^{2} \mathrm{~d} s\left(q_{i} \dot{q}_{i}^{2}+q_{i}^{2} \ddot{q}_{i}\right) \quad$ represents longitudinal inertia nonlinearity.

One should notice that the randomly excited system is an utterly different form excited by deterministic signals. For a 
harmonic signal excited cantilever, if the exciting frequency is very near the $i$-th mode's frequency, the internal resonance between the modes can be ignored. As is well known, the frequency of the colored noise distributes in a very wide range. Therefore, every mode may be under excitation when the basal motion is stochastic. However, the amplitude of the first mode predominates the amplitudes of other higher modes. In this paper, for simplicity, the contributions of higher modes to the total amplitude are ignored, and only the first vibration mode under colored noise excitation is studied.

By introducing the dimensionless transformation $\xi=$ $(s / L), x=(q / L), \eta=(\ddot{h} / L), \tau=(t / T), T=\left(1 / \lambda_{1}^{2}\right) \sqrt{\rho A L^{4} / E I}$, the dimensionless equation excited by a Gaussian colored noise was obtained as equation (4). For convenience, the dimensionless time $\tau$ is still written as $t$ in the following texture:

$$
\left(1+\alpha_{2} x^{2}\right) \ddot{x}+\omega^{2} x+\alpha_{1} x^{3}+\mu \dot{x}+\alpha_{2} \dot{x}^{2} x=\gamma \eta(t),
$$

where $\eta(t)$ is a Gaussian colored noise with a self-correlation function in the form of equation (1).

The coefficients of equation (4) are as follows:

$$
\begin{aligned}
& \omega^{2}=\frac{1}{\lambda_{1}^{4}} \int_{0}^{1} \phi_{1}^{\prime \prime 2} \mathrm{~d} \xi=1, \\
& \alpha_{1}=\frac{2}{\lambda_{1}^{4}} \int_{0}^{1} \phi_{1}^{\prime \prime 2} \phi_{1}^{\prime 2} \mathrm{~d} \xi=3.2713, \\
& \alpha_{2}=\int_{0}^{1}\left(\int_{0}^{\xi} \phi_{1}^{\prime 2} \mathrm{~d} \xi\right)^{2} \mathrm{~d} \xi=4.5968, \\
& \gamma=-\int_{0}^{1} \phi_{1} \mathrm{~d} \xi=0.7830, \\
& \mu=\frac{c L}{\lambda_{1}^{2} \sqrt{\rho A E I}} \int_{0}^{1} \varphi^{2} \mathrm{~d} \xi .
\end{aligned}
$$

At present, single dimension stochastic oscillator with only geometrical nonlinearity term $\alpha_{1} x^{3}$ has been studied sufficiently, but there is no existing method which can do stochastic averaging on this type of stochastic differential equation with both inertia nonlinearity and geometrical nonlinearity. In the following section, a new method is proposed to solve this problem.

\section{Methodology}

3.1. Definitions and Assumptions. Firstly, we defined the coordinate-dependent mass $M(x)=1+\alpha_{2} x^{2}$, stiffness term $g(x)=\omega^{2} x+\alpha_{1} x^{3}$, and the damping term $c(x, \dot{x})=$ $\mu \dot{x}+\alpha_{2} \dot{x}^{2} x$. Crucial presumptions are made here. $M(x)$ and $g(x)$ are strongly nonlinear terms, and the damping term $c(x, \dot{x})$ and the excitations $\eta(t)$ are weak.

The kinetic energy in equation (4) is

$$
T=\frac{1}{2} M(x) \dot{x}^{2}=\frac{1}{2}\left(1+\alpha_{2} x^{2}\right) \dot{x}^{2} .
$$

The potential energy of the conservative part of equation (4) is

$$
U(x)=\int g(x) \mathrm{d} x=\frac{1}{2} \omega^{2} x^{2}+\frac{1}{4} \alpha_{1} x^{4} .
$$

Thus, the Hamiltonian function is expressed as

$H(x, \dot{x})=T+U=\frac{1}{2}\left(1+\alpha_{2} x^{2}\right) \dot{x}^{2}+\frac{1}{2} \omega^{2} x^{2}+\frac{1}{4} \alpha_{1} x^{4}$.

Letting

$$
\begin{aligned}
& x=A \cos \theta, \\
& y=-A \nu \sin \theta, \\
& \theta=\Phi(t)+\tau(t),
\end{aligned}
$$

where $A$ is the stochastic amplitude and $\tau(t)$ is the stochastic phase. The stochastic frequency is

$$
\nu(A, \theta)=\frac{\mathrm{d} \Phi}{\mathrm{d} t} .
$$

Substituting equation (9) into equation (8), when $\theta=0$, the Hamiltonian function is

$$
H_{1}=\frac{1}{2} \omega^{2} A^{2}+\frac{1}{4} \alpha_{1} A^{4} .
$$

When $\theta \neq 0$, the Hamiltonian function is

$$
\begin{aligned}
H_{2}= & \frac{1}{2}\left(1+\alpha_{2} A^{2} \cos ^{2} \theta\right) A^{2} \nu^{2} \sin ^{2} \theta \\
& +\frac{1}{2} \omega^{2} A^{2} \cos ^{2} \theta+\frac{1}{4} \alpha_{1} A^{4} \cos ^{4} \theta .
\end{aligned}
$$

Obviously, at any time, the conservative Hamiltonian function remains constant, $H_{1}=H_{2}$, which yields

$$
\nu(A, \theta) \sin \theta=\sqrt{\frac{2[U(A)-U(A \cos \theta)]}{A^{2} M(A \cos \theta)} .}
$$

Equation (13) is crucial in the following deduction.

3.2. Transformations. Next, by dividing the coordinate-dependent mass $M(x)=1+\alpha_{2} x^{2}$ on each term of equation (4), equation (4) is transformed to be

$$
\left\{\begin{array}{l}
\dot{x}=y, \\
\dot{y}=-\frac{g(x)}{M(x)}-\frac{c(x, \dot{x})}{M(x)}+\frac{\gamma}{M(x)} \eta(t) .
\end{array}\right.
$$

Next, equation (14) can be transformed into a pair of equations about the stochastic transient equivalent amplitude $A$ and the stochastic phase $\tau(t)$.

Taking derivative to $x$ in equation (9) with respect to time $t$,

$$
\frac{\mathrm{d} A}{\mathrm{~d} t} \cos \theta-A \sin \theta\left(v+\frac{\mathrm{d} \tau}{\mathrm{d} t}\right)=y=-A v \sin \theta,
$$


which yields

$$
\frac{\mathrm{d} A}{\mathrm{~d} t} \cos \theta-A \sin \theta \frac{\mathrm{d} \tau}{\mathrm{d} t}=0
$$

Taking derivative to $y$ in equation (9) with respect to time $t$,

$$
\begin{aligned}
- & \frac{\mathrm{d} A}{\mathrm{~d} t} \nu \sin \theta-A \frac{\partial(\nu \sin \theta)}{\partial A} \times \frac{\mathrm{d} A}{\mathrm{~d} t}-A \frac{\partial(\nu \sin \theta)}{\partial \theta}\left(\nu+\frac{\mathrm{d} \tau}{\mathrm{d} t}\right) \\
= & -\frac{g(A \cos \theta)}{M(A \cos \theta)}-\frac{c(A \cos \theta,-A \nu \sin \theta)}{M(A \cos \theta)}+\frac{\gamma}{M(A \cos \theta)} \eta(t) .
\end{aligned}
$$

Equations (16) and (17) can be simplified to be a pair of stochastic differential equations represented by equations (18) and (19). The detailed procedure is displayed in Appendix:

$$
\begin{aligned}
\frac{\mathrm{d} A}{\mathrm{~d} t}= & \frac{c(A \cos \theta,-A \nu \sin \theta) \nu A \sin \theta}{g(A)}+\frac{A^{2} \nu^{3} \sin ^{2} \theta}{2 g(A)} \\
& \times \frac{\partial M(A \cos \theta)}{\partial \theta}-\frac{\gamma \times \eta(t) A \nu \sin \theta}{g(A)}, \\
\frac{\mathrm{d} \tau}{\mathrm{d} t}= & \frac{c(A \cos \theta,-A \nu \sin \theta) \nu \cos \theta}{g(A)}+\frac{A \nu^{3} \sin \theta \cos \theta}{2 g(A)} \\
& \times \frac{\partial M(A \cos \theta)}{\partial \theta}-\frac{\gamma \eta(t) \times \cos \theta \nu}{g(A)} .
\end{aligned}
$$

$$
\begin{aligned}
& m_{1}=\frac{c[A \cos \theta,-A \omega(A) \sin \theta] \omega(A) A \sin \theta}{g(A)}+\frac{A^{2} \omega^{3}(A) \sin ^{2} \theta}{2 g(A)} \times \frac{\partial M(A \cos \theta)}{\partial \theta}, \\
& m_{2}=\frac{c[A \cos \theta,-A \omega(A) \sin \theta] \omega(A) \cos \theta}{g(A)}+\frac{A \omega^{3}(A) \sin \theta \cos \theta}{2 g(A)} \times \frac{\partial M(A \cos \theta)}{\partial \theta}, \\
& b_{1}=-\frac{\gamma A \omega(A) \sin \theta}{g(A)} \\
& b_{2}=-\frac{\gamma \omega(A) \cos \theta}{g(A)} .
\end{aligned}
$$

Equation (21) is the standard form of stochastic averaging. $A(t)$ has been proved to converge weakly to a diffusive Markov process even in a semi-infinite time interval $[29,30]$. Thus, the Itô equation of the limiting diffusion process is of the following form:

$$
\mathrm{d} A=m(A) \mathrm{d} t+\sigma(A) \mathrm{d} W(t)
$$

where
3.3. Averaging on the Frequency. Next, before we do stochastic averaging on (18) and (19), the stochastic instantaneous frequency $\nu(A, \theta)$ should be averaged to the averaged frequency $\omega(A)$. The averaged frequency can be obtained using various methods, such as He's energy balance method [26], variational approach [27], and He's homotopy perturbation method (HPM) [28]. Here, He's energy balance method [14] is chosen to get the averaged frequency $\omega(A)$.

Taking $\theta=(\pi / 4)$ in equation (12), still considering $H_{1}=H_{2}$, the $\omega(A)$ can be solved out as follows:

$$
\omega(A)=\frac{\sqrt{3 A^{2} \alpha_{1}+4 \omega^{2}}}{\sqrt{4+2 A^{2} \alpha_{2}}} .
$$

Thus, the stochastic instantaneous frequency $\nu(A, \theta)$ can be displaced to be the averaged frequency $\omega(A)$ in equations (18) and (19). This approach has been proved to be effective in dealing with single-degree-of-freedom strongly nonlinear stochastic equations by $\mathrm{Ge}$ [23].

3.4. Stochastic Averaging Procedure. With the transformations accomplished, equations (18) and (19) can be written as

$$
\begin{gathered}
\frac{\mathrm{d} A}{\mathrm{~d} t}=m_{1}(A, \tau)+b_{1}(A, \tau) \eta(t), \\
\frac{\mathrm{d} \tau}{\mathrm{d} t}=m_{2}(A, \tau)+b_{2}(A, \tau) \eta(t),
\end{gathered}
$$

where

$$
\begin{aligned}
m(A) & =\left\langle m_{1}+\int_{0}^{+\infty}\left(\left.\left.\frac{\partial b_{1 i}}{\partial A}\right|_{t} b_{1 j}\right|_{t+\tau}+\left.\left.\frac{\partial b_{1 i}}{\partial \tau}\right|_{t} b_{2 j}\right|_{t+\tau}\right) R_{i j}(\tau) \mathrm{d} \tau\right\rangle \\
\sigma^{2}(A) & =\left\langle\int_{-\infty}^{+\infty}\left(\left.\left.b_{1 i}\right|_{t} b_{1 j}\right|_{t+\tau}\right) R_{i j}(\tau) \mathrm{d} \tau\right\rangle,
\end{aligned}
$$

where $W(t)$ is the standard unit Brownian motion, $\langle\cdot\rangle$ is the averaging with respect to time, and $R_{i j}(\tau)$ is the correlation 
function shown as equation (1). The obtained drift coefficient $m(A)$ and the diffusion coefficient $\sigma^{2}(A)$ are as follows:

$$
m(A)=\frac{a_{10} A^{10}+a_{8} A^{8}+a_{6} A^{6}+a_{4} A^{4}+a_{2} A^{2}+a_{0}}{4 A\left(2+A^{2} \alpha_{2}\right)\left(A^{2} \alpha_{1}+\omega^{2}\right)^{3}\left[3 A^{2} \alpha_{1}+4 \omega^{2}+2\left(2+A^{2} \alpha_{2}\right) \lambda^{2}\right]},
$$

where

$$
\begin{aligned}
a_{10} & =-6 \alpha_{1}^{3} \alpha_{2} \mu-9 \alpha_{1}^{4} \mu, \\
a_{8} & =-12 \alpha_{1}^{3} \mu \lambda^{2}-20 \alpha_{1}^{2} \alpha_{2} \mu \omega^{2} \lambda^{2}-42 \alpha_{1}^{3} \lambda^{2} \mu \omega^{2}, \\
a_{6} & =-40 \alpha_{1}^{2} \mu \omega^{2} \lambda^{2}-22 \alpha_{1} \alpha_{2} \mu \omega^{4} \lambda^{2}-73 \alpha_{1}^{2} \mu \omega^{4}-6 \alpha_{1}^{2} \alpha_{2} \gamma^{2} D \lambda^{2}, \\
a_{4} & =-44 \alpha_{1} \mu \omega^{4} \lambda^{2}-8 \alpha_{2} \mu \omega^{6} \lambda^{2}-56 \alpha_{1} \mu \omega^{6}-10 \alpha_{1} \alpha_{2} \gamma^{2} \omega^{2} D \lambda^{2}, \\
a_{2} & =-16 \mu \omega^{6}-16 \lambda^{2} \mu \omega^{6}+8 \alpha_{1} \gamma^{2} \omega^{2} D \lambda^{2}, \\
a_{0} & =16 \gamma^{2} \omega^{4} D \lambda^{2}, \\
\sigma^{2}(A) & =\frac{\gamma^{2}\left(3 A^{2} \alpha_{1}+4 \omega^{2}\right) D \lambda^{2}}{\left(A^{2} \alpha_{1}+\omega^{2}\right)^{2}\left[3 A^{2} \alpha_{1}+4 \omega^{2}+2\left(2+A^{2} \alpha_{2}\right) \lambda^{2}\right]} .
\end{aligned}
$$

The averaged Fokker-Planck-Kolmogorov (FPK) equation associated with Itô equation (23) is of the following form [30, 31]:

$$
\frac{\partial p}{\partial t}=-[m(A)] \frac{\partial p}{\partial A}+\left[\frac{1}{2} \sigma^{2}(A)\right] \frac{\partial^{2} p}{\partial A^{2}},
$$

where $p(A, t)$ is the transition probability density of displacement amplitude. A nontrivial stationary solution exists due to nonvanishing external excitation. Under assumption of zero probability flow at the two boundaries $A=0$ and $A=\infty$, the stationary solution of FPK equation (27) [31] for system (23) is of the following form:

$$
p(A)=\frac{N}{\sigma^{2}(A)} e^{\left[2 \int\left(m(A) / \sigma^{2}(A)\right) \mathrm{d} A\right]},
$$

where constant $N$ is the normalization constant. Substituting equations (25) and (26) into equation (28),

$$
\begin{aligned}
P(A)= & N \frac{A\left(A^{2} \alpha 1+\omega^{2}\right)\left[3 A^{2} \alpha_{1}+4 \omega^{2}+2\left(2+A^{2} \alpha_{2}\right) \lambda^{2}\right]}{\sqrt{3 A^{2} \alpha_{1}+4 \omega^{2}}}\left(2+A^{2} \alpha_{2}\right)^{(1 / 2)\left[-1+\left(6 \alpha_{1}^{2}-7 \alpha_{1} \alpha_{2} \omega^{2}+2 \alpha_{2}^{2} \omega^{4} / \alpha_{2}^{3} \gamma^{2} D \lambda^{2}\right)\right]} \\
& \cdot e^{\left[\left(A^{4}\left(-3 \alpha_{1}^{2} \alpha_{2} \mu-2 \alpha_{1} \alpha_{2}^{2} \mu \lambda^{2}\right)+A^{2}\left(12 \alpha_{1}^{2} \mu-14 \alpha_{1} \alpha_{2} \mu \omega^{2}-4 \alpha_{2}^{2} \mu \omega^{2} \lambda^{2}\right) / 8 \alpha_{2}^{2} \gamma^{2} D \lambda^{2}\right)\right]} .
\end{aligned}
$$

The stationary probability density of total energy $H$ or energy envelope can be obtained as follows:

$$
p(H)=p(A)\left|\frac{\mathrm{d} A}{\mathrm{~d} H}\right|=\left.\frac{p(A)}{g(A)}\right|_{A=g^{-1}(H)},
$$

where $A=g^{-1}(H)=\sqrt{-\left(\omega^{2} / \alpha_{1}\right)+\left(\sqrt{4 H \alpha_{1}+\omega^{4}} / \alpha_{1}\right)}$. It is solved from equation (11).
The joint stationary probability density of displacement $x$ and velocity $y$ can be further obtained from $p(H)$ as follows:

$$
p(x, y)=\left.\frac{p(H)}{T(H)}\right|_{H=(1 / 2) \omega^{2} x^{2}+(1 / 4) \alpha_{1} x^{4}+(1 / 2)\left(1+\alpha_{2} x^{2}\right) y^{2},}
$$

where $T(H)$ can be obtained from $T(H)=2 \pi /\left.\omega(A)\right|_{A=g^{-1}(H)}$. 


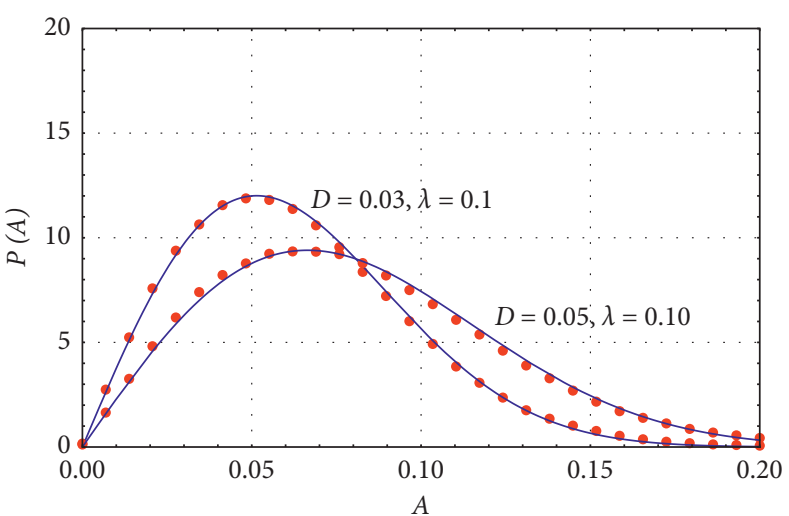

(a)

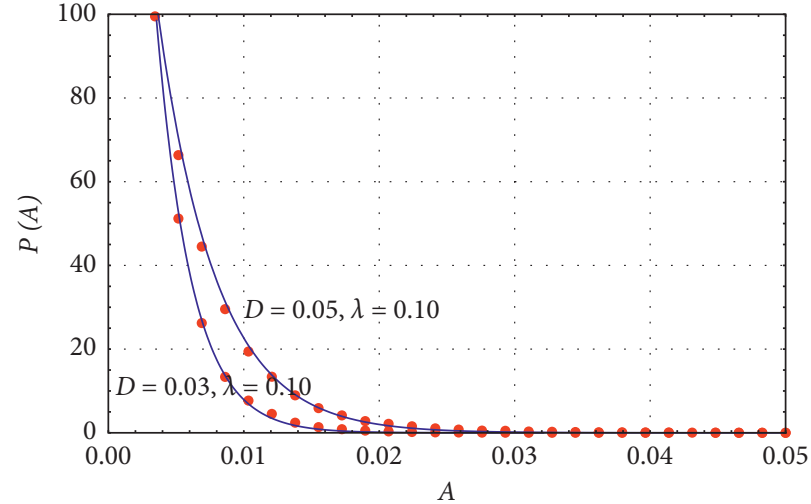

(b)

FIGURe 2: Stationary probability density of displacement amplitude and Hamilton: (a) PDF of amplitude A; (b) PDF of Hamiltonian function $\mathrm{H}$ (solid lines: stochastic averaging equations (29) and (30); dots: Monte Carlo simulation on equation (6)).

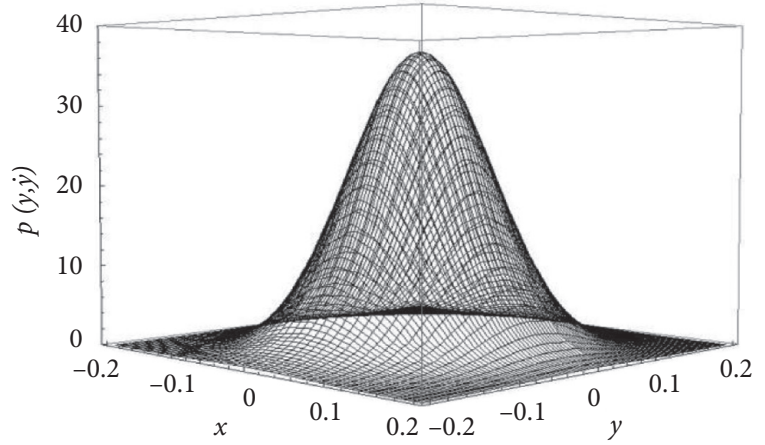

(a)

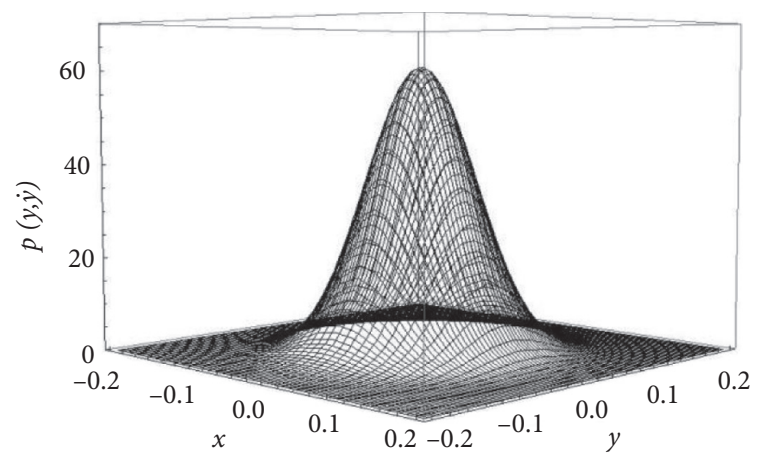

(c)

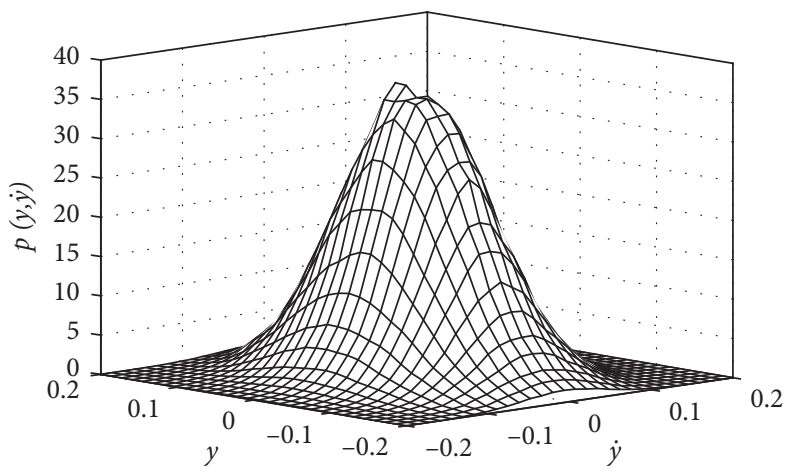

(b)

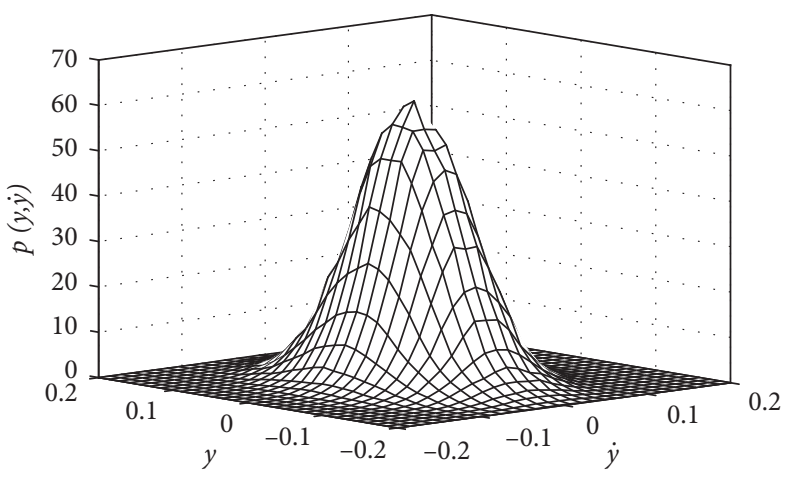

(d)

FIGURE 3: Stationary joint probability density $\lambda=0.1$ : (a) $D=0.05$, stochastic averaging equation (31); (b) $D=0.05$, Monte Carlo simulation; (c) $D=0.03$, stochastic averaging equation (31); (d) $D=0.03$, Monte Carlo simulation.

If one take the velocity $y=0$ in equation (31), the stationary PDF of displacement $x$ is obtained.

\section{Numerical Simulations}

One precondition of applying the stochastic averaging procedure in Section 3 is that the damping and the noise strength are all small enough compared with the conservative energy so that damping coefficient and the densities of the noise are chosen as weak ones in order to ensure that the energy dissipated by damping can balance the input energy induced by the noise. The densities of white noise are set as $D=0.05$ and $D=0.03$, and the dimensionless damping coefficient is set as $\mu=0.1$. Another precondition of applying the stochastic averaging procedure is that the noise must be wideband noise. As seen from Figure 1 and equation (1), the larger the correlation time parameter is, and the narrower the noise band becomes. In this article, the correlation time parameter was set as $\lambda=0.1$ to ensure that the excitation is wideband. For each density, totally 2000 sets of colored noise 


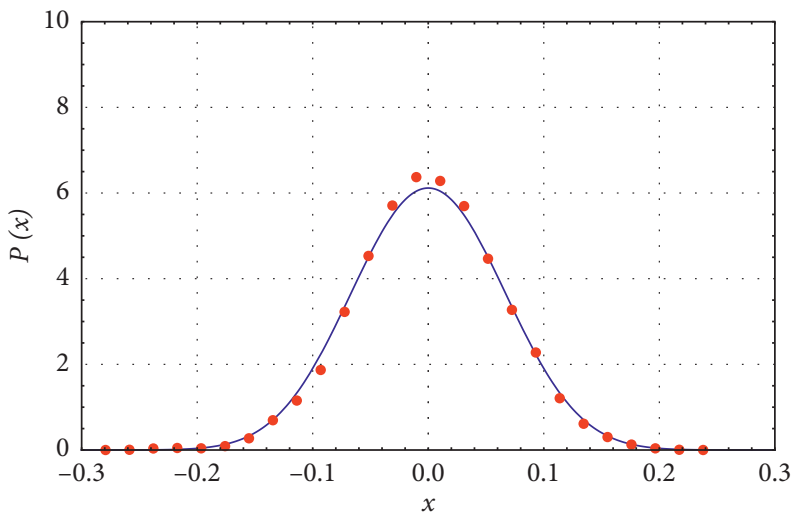

FIgURE 4: Stationary probability density of displacement $x(D=0.03$ and $\lambda=0.1)$ (solid lines: stochastic averaging; dots: Monte Carlo simulation on equation (6)).

are imposed to the system (6). Each set noise includes 40000 numbers. The dimensionless time step is set as $\Delta t=0.005$. The numerical solutions of the oscillator equation (6) could be obtained by an order-2 stochastic Runge-Kutta algorithm [24]. The last 10000 steps are kept as the steady-state responses. For each step, the transient Hamiltonian function $H$ value is calculated out; simultaneously, the transient amplitude $A=\sqrt{-\left(\omega^{2} / \alpha_{1}\right)+\left(\sqrt{4 H \alpha_{1}+\omega^{4}} / \alpha_{1}\right)}$ is solved.

Finally, doing statistic on total stationary $n=2000 \times 10000$ values of $H$ and $A$, the stationary probability density function (PDF) of the amplitude is shown in Figure 2(a), and the stationary PDF of the energy is shown in Figure 2(b). Subsequently, the joint PDF of displacement $x$ and velocity $y$ is numerically simulated. We take $40 \times 40$ grids in a range $\{-0.2 \leq x \leq 0.2,-0.2 \leq y \leq 0.2\}$ with a space gap $\Delta x=\Delta y=0.01$. Then, we count the numbers $n_{i j}(i, j=1 \sim 40)$ of the $n=2000 \times 10000$ stationary $(x, y)$ dots which fall within each grid. Finally, the joint $\operatorname{PDF} P(x, y)$ value of each grid can be calculated out $\operatorname{by} P\left(x_{i}, y_{i}\right)=$ $\left(n_{i j} /(n \times \Delta x \times \Delta y)\right)$. The joint PDFs are illustrated in Figure 3. From Figures 2 and 3, we found the theoretical prediction by the new stochastic averaging method deduced in this paper coincides with the digital Monte Carlo method. Figure 4 is the stationary PDF of the displacement $x$.

\section{Conclusion}

A new stochastic averaging method for solving the cantilever beam model with both inertia nonlinearity and geometric nonlinearity which is excited by colored noise is proposed in this paper. The old method is extended to a new filed. The key issue of this method is balancing the input energy and diffusion energy of the Hamiltonian function.
Expressions for the drift coefficient and diffusion coefficient in the Itô function are obtained by the averaging procedure. The probability of stationary responses of the amplitude, the Hamiltonian function, the stationary PDF of displacement $x$, and the joint probability of displacement and velocity are studied. The analytical approximations are consistent with the Monte Carlo numerical simulation. Therefore, the presented approach is effective to solve the equations for single-degree-of-freedom (SDOF) oscillators with inertia and curvature coexisting nonlinearities.

\section{Appendix}

This appendix is a detailed deduction procedure to transform equations (16) and (17) to be equations (18) and (19). It is obvious that equation (17) contains two important expressions $(\partial(\nu \sin \theta) / \partial A)$ and $(\partial(\nu \sin \theta) / \partial \theta)$ which can be gained by taking partial derivative to equation (13).

Taking partial derivative to equation (13) with respect to amplitude $A$, one obtains (A.1).

Substituting the expression

$$
v(A, \theta) A \sin \theta=\sqrt{\frac{2[U(A)-U(A \cos \theta)]}{M(A \cos \theta)}},
$$

which is obvious in equation (13), into equation (A.1) yields

$$
\begin{aligned}
\frac{\partial(\nu \sin \theta)}{\partial A}= & \frac{g(A)-g(A \cos \theta) \cos \theta}{\nu A^{2} \sin \theta M(A \cos \theta)}-\frac{\nu \sin \theta}{A} \\
& -\frac{\nu \sin \theta}{2 M(A \cos \theta)} \times \frac{\partial M(A \cos \theta)}{\partial A} .
\end{aligned}
$$

Similarly, taking partial derivative to equation (13) with respect to time $\theta$, one obtains

$$
\begin{aligned}
\frac{\partial(\nu \sin \theta)}{\partial \theta}= & \sqrt{2}\left\{\frac{1}{2 \sqrt{U(A)-U(A \cos \theta)}} \times \frac{[-g(A \cos \theta)](-A \sin \theta)}{A \sqrt{M(A \cos \theta)}}\right. \\
& \left.-\frac{\sqrt{U(A)-U(A \cos \theta)}}{2 A} \times \frac{1}{M(A \cos \theta)^{3 / 2}} \times \frac{\partial M(A \cos \theta)}{\partial \theta}\right\}
\end{aligned}
$$


Substituting $\sqrt{(2[U(A)-U(A \cos \theta)] / M(A \cos \theta))}$ into equation (A.3) yields

$\frac{\partial(\nu \sin \theta)}{\partial \theta}=\frac{g(A \cos \theta)}{A \nu M(A \cos \theta)}-\frac{\nu \sin \theta}{2 M(A \cos \theta)} \times \frac{\partial M(A \cos \theta)}{\partial \theta}$.
Substituting equations (A.2) and (A.4) into equation (17), one obtains

By doing Eq. $(A .5) \times A \sin \theta+$ Eq. $(15) \times[(g(A \cos \theta) / \nu M$ $(A \cos \theta))-(\nu A \sin \theta / 2 M(A \cos \theta)) \times(\partial M(A \cos \theta) / \partial \theta)]$, one finds

$$
\left\{\begin{array}{l}
\frac{\mathrm{d} A}{\mathrm{~d} t}\left\{\frac{g(A)-g(A \cos \theta) \cos \theta}{\nu A \sin \theta M}-\frac{\nu A \sin \theta}{2 M(A \cos \theta)} \times \frac{\partial M(A \cos \theta)}{\partial A}\right\} \\
+\frac{\mathrm{d} \tau}{\mathrm{d} t}\left\{\frac{g(A \cos \theta)}{\nu M(A \cos \theta)}-\frac{A \nu \sin \theta}{2 M(A \cos \theta)} \times \frac{\partial M(A \cos \theta)}{\partial \theta}\right\} \\
=\frac{c(A \cos \theta,-\nu A \sin \theta)}{M(A \cos \theta)}+\frac{A \nu^{2} \sin \theta}{2 M(A \cos \theta)} \times \frac{\partial M(A \cos \theta)}{\partial \theta}-\frac{\gamma \eta(t)}{M(A \cos \theta)}
\end{array}\right.
$$

Considering

$$
\begin{aligned}
& \frac{\partial M(A \cos \theta)}{\partial A}=2 \alpha_{2} A \cos ^{2} \theta, \\
& \frac{\partial M(A \cos \theta)}{\partial \theta}=-2 \alpha_{2} A^{2} \sin \theta \cos \theta,
\end{aligned}
$$

we have

$$
\begin{aligned}
& -\frac{v A^{2} \sin ^{2} \theta}{2 M(A \cos \theta)} \times \frac{\partial M(A \cos \theta)}{\partial A}=\frac{-v A^{3} \alpha_{2} \sin ^{2} \theta \cos ^{2} \theta}{M(A \cos \theta)}, \\
& \frac{v A \sin \theta \cos \theta}{2 M(A \cos \theta)} \times \frac{\partial M(A \cos \theta)}{\partial \theta}=\frac{\nu A^{3} \alpha_{2} \sin ^{2} \theta \cos ^{2} \theta}{M} .
\end{aligned}
$$

Substituting equations (A.8) and (A.9) into equation (A.6) yields equation (A.10):

$$
\begin{aligned}
\frac{\mathrm{d} A}{\mathrm{~d} t}= & \frac{c(A \cos \theta,-A \nu \sin \theta) \nu A \sin \theta}{g(A)}+\frac{A^{2} \nu^{3} \sin ^{2} \theta}{2 g(A)} \\
& \times \frac{\partial M(A \cos \theta)}{\partial \theta}-\frac{\gamma \eta(t) A \nu \sin \theta}{g(A)} .
\end{aligned}
$$

Substituting equation (18) into equation (19), one gains equation (A.11).

$$
\begin{aligned}
\frac{\mathrm{d} \tau}{\mathrm{d} t}= & \frac{c(A \cos \theta,-A \nu \sin \theta) \nu \cos \theta}{g(A)}+\frac{A \nu^{3} \sin \theta \cos \theta}{2 g(A)} \\
& \times \frac{\partial M(A \cos \theta)}{\partial \theta}-\frac{\gamma \eta(t) \cos \theta \nu}{g(A)} .
\end{aligned}
$$

\section{Data Availability}

All data generated or analyzed during this study are included in this published article, and other pieces of information are available from the corresponding author on reasonable request.

\section{Conflicts of Interest}

The authors declare that they have no conflicts of interest.

\section{Acknowledgments}

The authors gratefully acknowledge the support of the Program for Innovative Research Team in University of 
Tianjin (Grant no. TD13-5037), Natural Science Foundation of China (Grant no. 11402186), and Natural Science Foundation of Tianjin (Grant nos. 14JCQNJC05600 and 18JCQNJC05300).

\section{References}

[1] A. H. Nayfeh and P. F. Pai, "“'Non-linear non-planar parametric responses of an inextensional beam," International Journal of Non-linear Mechanics, vol. 24, no. 17, pp. 139-158, 1989.

[2] A. H. Nayfeh and S. A. Nayfeh, "Nonlinear normal modes of a continuous system with quadratic nonlinearities," Journal of Vibration and Acoustics, vol. 117, no. 2, pp. 199-205, 1995.

[3] S. K. Dwivedy and R. C. Kar, "'Nonlinear response of a parametrically excited system using higher-order method of multiple scales," Nonlinear Dynamics, vol. 20, no. 2, pp. 115-130, 1999.

[4] H. Yabuno and A. H. Nayfeh, "“'Nonlinear normal modes of a parametrically excited cantilever beam," Normal Modes and Localization in Nonlinear Systems, vol. 25, pp. 65-77, 2001.

[5] S. K. Dwivedy and R. C. Kar, "'Nonlinear dynamics of a cantilever beam carrying an attached mass with 1:3:9 internal resonances," Nonlinear Dynamics, vol. 31, no. 1, pp. 49-72, 2003.

[6] M. N. Hamdan, A. A. Al-Qaisia, and B. O. Al-Bedoor, "Comparison of analytical techniques for nonlinear vibrations of a parametrically excited cantilever," International Journal of Mechanical Sciences, vol. 43, no. 6, pp. 1521-1542, 2001.

[7] C. J. Silva and M. F. Daqaq, "Nonlinear flexural response of a slender cantilever beam of constant thickness and linearlyvarying width to a primary resonance excitation," Journal of Sound and Vibration, vol. 389, pp. 438-453, 2017.

[8] L. D. Zavodney and A. H. Nayfeh, "The non-linear response of a slender beam carrying a lumped mass to a principal parametric excitation: theory and experiment," International Journal of Non-linear Mechanics, vol. 24, no. 2, pp. 105-125, 1989.

[9] H. Yabuno, Y. Ide, and N. Aoshima, "Nonlinear analysis of a parametrically excited cantilever beam. (Effect of the tip mass on stationary response)," JSME International Journal Series C, vol. 41, no. 3, pp. 555-562, 1998.

[10] G. K. Er and V. P. Iu, The Probabilistic Solutions of the Cantilever Excited by Lateral and Axial Excitations Being Gaussian White Noise, pp. 257-270, Springer, Cham, Switzerland, 2014.

[11] Z. H. Feng and H. Y. Hu, "Largest lyapunov exponent and almost certain stability analysis of slender beams under a large linear motion of basement subject to narrowband parametric excitation," Journal of Sound and Vibration, vol. 257, no. 4, pp. 733-752, 2002.

[12] Z. H. Feng, X. J. Lan, and X. D. Zhu, "Principal parametric resonances of a slender cantilever beam subject to axial narrow-band random excitation of its base," International Journal of Non-linear Mechanics, vol. 42, no. 10, pp. 11701185, 2007.

[13] Z. H. Feng, X. J. Lan, and X. D. Zhu, "Explanation on the importance of narrow-band random excitation characters in the response of a cantilever beam," Journal of Sound and Vibration, vol. 325, no. 4-5, pp. 923-937, 2009.

[14] Z. H. Feng, X. D. Zhu, and X. J. Lan, "Stochastic jump and bifurcation of a slender cantilever beam carrying a lumped mass under narrow-band principal parametric excitation,"
International Journal of Non-linear Mechanics, vol. 46, no. 10, pp. 1330-1340, 2011.

[15] G. Ge and W. Yan, "Cantilever model with curvature nonlinearity and longitudinal inertia excited by lateral basal moments being Gaussian white noise," Journal of Vibroengineering, vol. 20, no. 1, pp. 677-690, 2018.

[16] P. S. Landau and R. L. Stratonovich, Theory of stochastic transitions of various systems between different states, pp. 63-45, Moscow University, Richland Center, WI, USA, 1962.

[17] R. Z. Khasminskii, "On the behaviour of a conservative system with small friction and small random noise," Journal of Applied Mathematics and Mechanics, vol. 28, pp. 1126-1130, 1964.

[18] W. Q. Zhu, "Stochastic averaging of the energy envelope of nearly lyapunov systems," random vibrations and reliability," in Proceedings of the I U TAM Symposium, pp. 347-357, Akademie, Berlin, Germany, 1983.

[19] W. Zhu and Y. K. Lin, "Stochastic averaging of energy envelope," Journal of Engineering Mechanics, vol. 117, no. 8, pp. 1890-1905, 1991.

[20] Z. L. Huang, W. Q. Zhu, and Y. Suzuki, "Stochastic averaging of strongly non-linear oscillators under combined harmonic and white-noise excitations," Journal of Sound and Vibration, vol. 238, no. 2, pp. 233-256, 2000.

[21] W. Q. Zhu, Z. L. Huang, and Y. Suzuki, "Response and stability of strongly non-linear oscillators under wide-band random excitation," International Journal of Non-linear Mechanics, vol. 36, no. 8, pp. 1235-1250, 2001.

[22] Z. Xu and Y. K. Cheung, "Averaging method using generalized harmonic functions for strongly non-linear oscillators," Journal of Sound and Vibration, vol. 174, no. 4, pp. 563-576, 1994.

[23] G. Ge and Z. Li, "A modified stochastic averaging method on single-degree-of-freedom strongly nonlinear stochastic vibrations," Chaos, Solitons \& Fractals, vol. 91, pp. 469-477, 2016.

[24] R. L. Honeycutt, "Stochastic Runge-Kutta algorithms. II. Colored noise," Physical Review A, vol. 45, no. 2, pp. 604-610, 1992.

[25] Y. Xu, R. Gu, H. Zhang et al., "“Stochastic bifurcations in a bistable Duffing-Van der Pol oscillator with colored noise,", Physical Review E, vol. 83, no. 5, pp. 056215 1-7, 2011.

[26] J. H. He, "“Preliminary report on the energy balance for nonlinear oscillations," Mechanics Research Communications, vol. 29, no. 2-3, pp. 107-111, 2002.

[27] J.-H. He, "Some asymptotic methods for strongly nonlinear equations," International Journal of Modern Physics B, vol. 20, no. 10, pp. 1141-1199, 2006.

[28] Y. Wu and J.-H. He, "Homotopy perturbation method for nonlinear oscillators with coordinate-dependent mass," Results in Physics, vol. 10, pp. 270-271, 2018.

[29] W. Q. Zhu, "“Recent developments and applications of the stochastic averaging method in random vibration," Applied Mechanics Reviews, vol. 49, no. 10S, pp. 72-80, 1996.

[30] J. B. Roberts and P. D. Spanos, "Stochastic averaging: an approximate method of solving random vibration problems," International Journal of Non-linear Mechanics, vol. 21, no. 2, pp. 111-134, 1986.

[31] A. T. Fuller, "Analysis of nonlinear stochastic systems by means of the Fokker-Planck equation $\dagger$," International Journal of Control, vol. 9, no. 6, pp. 603-655, 1969. 